\title{
Isolated Oculomotor Cranial Nerve Palsy as Unusual Presentation of Primary High-Grade Burkitt's Cell Lymphoma in HIV Patient: Case Report and Literature Review
}

\author{
Elabbass A. Abdelmahmuod ${ }^{a}$ Mohammed Abdulgayoom $^{a}$ \\ Mohammed Elhadi ${ }^{a}$ Hiba Magboul ${ }^{a}$ Mouhammad Z. Sharaf Eldean ${ }^{b}$ \\ Sali Alatasi ${ }^{b}$ \\ aDepartment of Internal Medicine, Hamad Medical Corporation, Doha, Qatar; ${ }^{b}$ Department \\ of pathology, Hamad Medical Corporation, Doha, Qatar
}

Keywords

Oculomotor · Third cranial nerve · B-cell · Lymphoma $\cdot$ Burkitt's

\begin{abstract}
Burkitt lymphoma (BL) is a highly aggressive B-cell neoplasm that is well known to be associated with HIV. The presentation usually reflects the underline immunodeficiency state (like opportunistic infections and chronic diarrhea, and enlarged lymph nodes). The most common causes for 3rd cranial nerve palsy are intracranial aneurysm, ischemia, trauma, and migraine. But for our case, it turned out to be associated with underline HIV and BL, which is an unusual cause. Here, we present a 43-year-old gentleman with no past medical history presented to the emergency department with 4 days history of drooping of left eyelid and headache and binocular diplopia with no other neurological features. CT abdomen showed lymph node enlargement. Lymph node biopsy showed the characteristic of Burkitt's cell lymphoma. He was started on chemotherapy, but unfortunately, he died. We're enlightening this case of an isolated oculomotor nerve palsy to diagnose lethal pathology like disseminated BL.
\end{abstract}




\section{Introduction}

HIV patients have a significantly increased risk of cancer, with approximately $25-40 \%$ of them developing a malignancy in their lives [1]. Ten percent developing non-Hodgkin lymphoma (NHL) expressly, from this Burkitt lymphoma (BL) represent approximately 25\% of NHL in HIV patients [2].

BL is a highly aggressive B-cell neoplasm that can present in one of 3 distinct clinical forms: endemic, sporadic, and immunodeficiency associated. Although they have the same histological picture and similar clinical behavior, there are differences in epidemiology, clinical presentation, and genetic features between the 3 forms [3].

In general, patients with BL present with rapidly growing tumor masses and often have tumor lysis evidence. The endemic form, which is found in equatorial Africa, presents a jaw or facial bone tumor that spreads to extranodal sites. In contrast, the nonendemic (sporadic) form, seen in the USA and Western Europe, has an abdominal presentation, most often with the massive disease. Immunodeficiency-related cases more often involve lymph nodes [4].

The presentation of patients with immunodeficiency-related BL is often accompanied by signs or symptoms related to the underlying immunodeficiency (e.g., AIDS, congenital immunodeficiency, acquired immunodeficiency due to hematopoietic or solid organ transplantation). Immunodeficiency-related cases more often involve lymph nodes, bone marrow, and central nervous system (CNS) [5].

A third cranial nerve palsy can result from lesions anywhere along its path between the oculomotor nucleus in the midbrain and the orbit's extraocular muscles. The most common causes of 3rd cranial nerve palsy are intracranial aneurysm, ischemia, trauma, and migraine [6]. Here, we are reporting a middle-aged male case presented with isolated 3rd cranial nerve palsy, which turned to be associated with underline HIV infection, and BL, which is an unusual presentation for both.

\section{Case Presentation}

A 43-year-old gentleman with no past medical history presented to the emergency department with 4 days history of drooping of the left eyelid and headache and binocular diplopia (horizontal and vertical) with no other neurological symptoms. On examination, he had unequal pupils, left more prominent than the right ( $5 \mathrm{vs.} 3 \mathrm{~mm}$ ), abducted and down deviated left eye with complete ptosis, no facial asymmetry, preserved sensation over the face, no motor and sensory deficit. Seen by ophthalmology and confirmed left oculomotor palsy. Seen by neurology and requested a plain CT head and angiogram to rule out compression and posterior communicating aneurysm, both ruled out. Further evaluation was done by MRI head and orbit, which were unremarkable.

During the hospital course, he developed epigastric pain aggravated by food; the abdominal ultrasound was done for further evaluation and showed suspicion of bowel wall thickening. For further evaluation, CT abdomen was done and showed jejunal wall thickening and irregular outline with multiple enlarged lymph nodes seen in the bilateral inguinal.

CT chest was requested to look for mediastinal lymph node and showed a large filling defect present in the right atrium extended into the upper end of the inferior vena cava, findings suggestive of lymphoma. Further evaluation was requested based on the above findings and include: ECHO and cardiac MRI showed an intracardiac mass consistent with lymphoma. Lymph node biopsy was done (see Fig. 1-5), which showed features consistent with BL.

\section{Karger'}




\section{Case Reports in Oncology}

\begin{tabular}{l|l}
\hline Case Rep Oncol 2021;14:1555-1560 \\
\hline DOI: 10.1159/000514983 & $\begin{array}{l}\text { @ 2021 The Author(s). Published by S. Karger AG, Basel } \\
\text { www.karger.com/cro }\end{array}$ \\
\hline
\end{tabular}

Abdelmahmuod et al.: Oculomotor Cranial Nerve Palsy with Burkitt's Lymphoma

Fig. 1. Showed dermis is infiltrated by sheets of intermediate-sized lymphocytes.

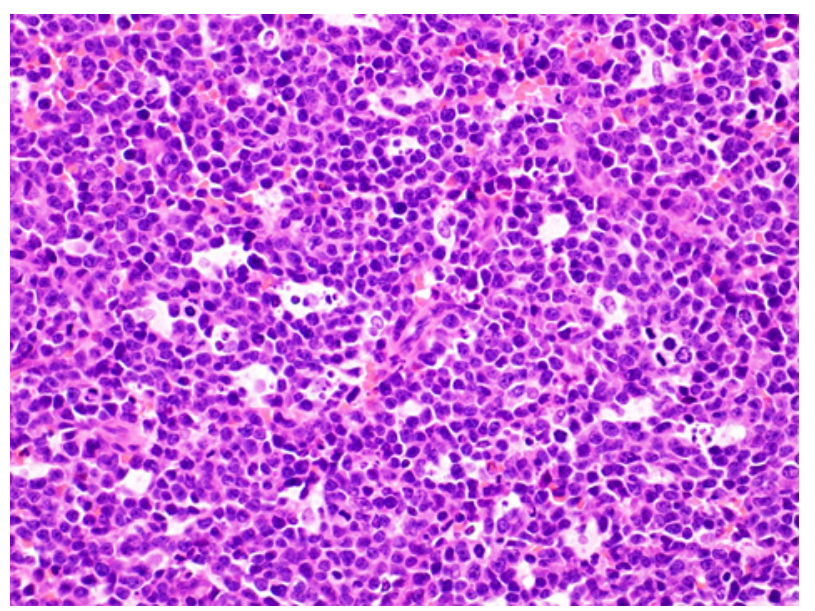

Fig. 2. Showed CD20 immunohistochemical stain is positive.

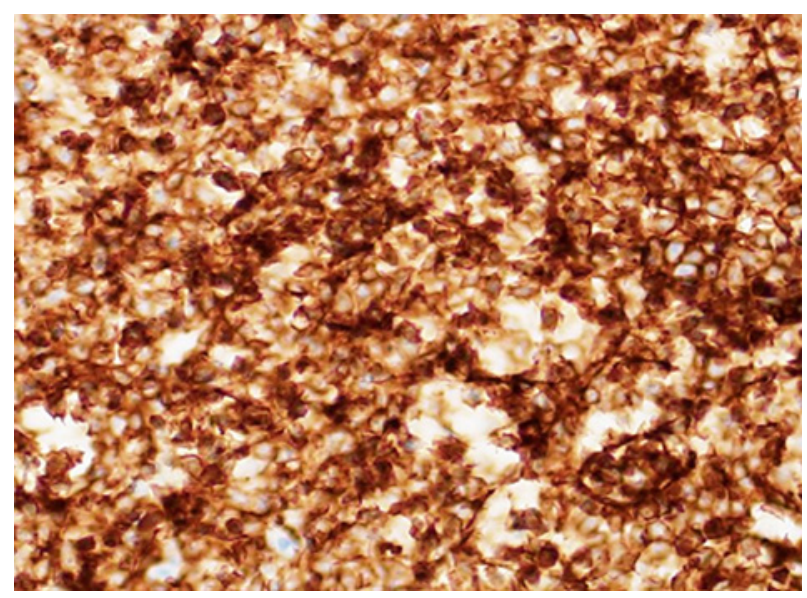

Fig. 3. Showed the tumor cells are positive for CD10 immunohistochemical stain.

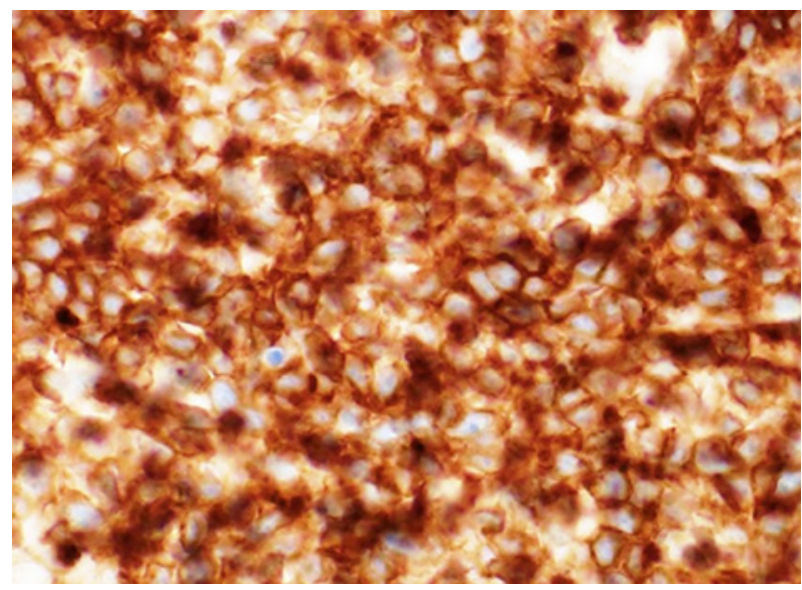

He was shifted to a hematology/oncology hospital to receive chemotherapy; he initially received $\mathrm{CHOP}$ protocol (cyclophosphamide, doxorubicin, vincristine, and prednisolone). He received intrathecal methotrexate; initially, he showed a response, but unfortunately, he deteriorated and died. 


\section{Case Reports in Oncology}

Case Rep Oncol 2021;14:1555-1560

Fig. 4. Neoplastic cells are positive for C-MYC.

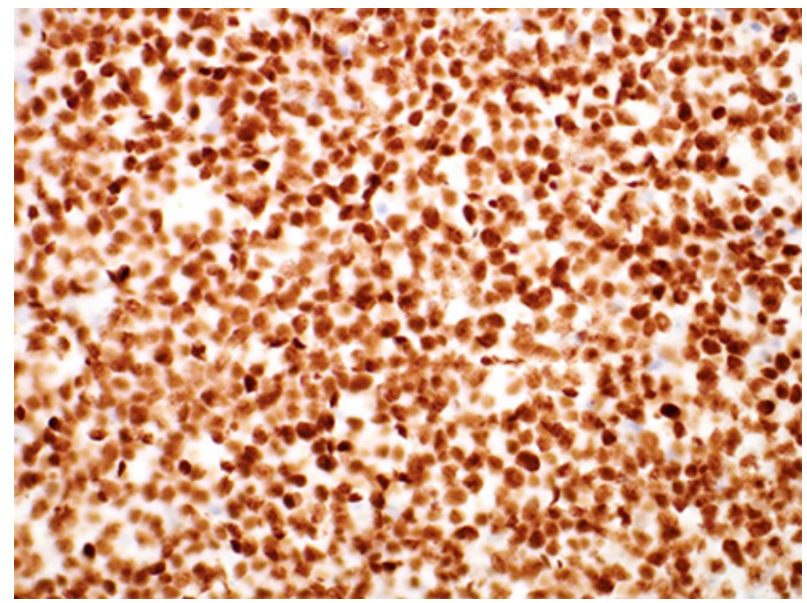

Fig. 5. Showed in situ hybridization for EBER.

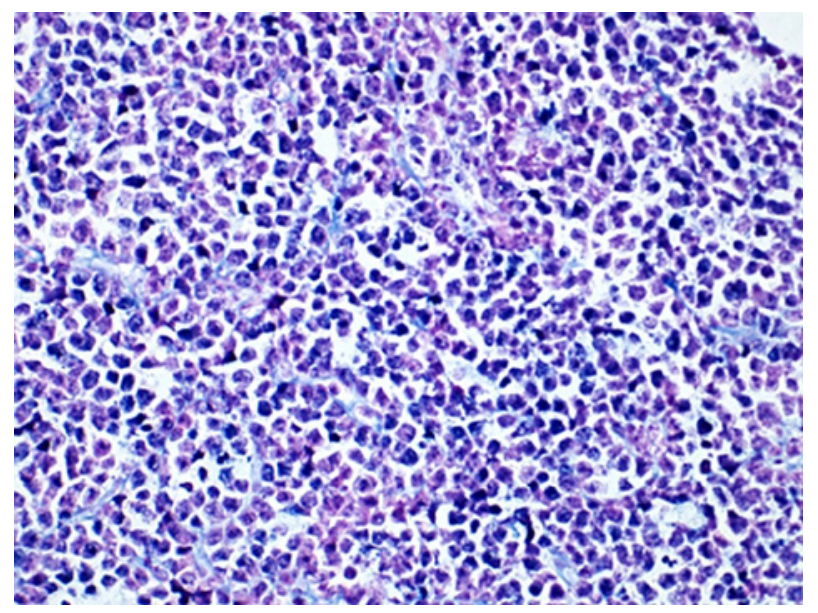

\section{Discussion}

NHL is a malignant disease that initiates in the lymphatic system and can spread to the CNS, recognized as secondary CNS lymphoma (SCNSL). Leptomeninges are most frequently involved in SCNSL and can manifest with cranial nerve involvement [7].

NHL metastasis that involves CNS frequently contributes to significant impairment with neurology and limited life expectancy. Approximately, 5-10\% of systemic NHL patients will have SCNSL [8].

Neurolymphomatosis has been defined as the infiltration of peripheral nerves, nerve roots, and cranial nerves by lymphomatosis and frequently characterized by painful or painless polyneuropathy or mononeuropathy of the periphery [9].

Cranial nerve dysfunction may result from primary or secondary lymphoma, and the most common cranial nerve involved due to leptomeningeal disease is the facial nerve [10]. Ocular presentations of SCNSL are unusual and involve optic nerve lesions, nystagmus, ptosis, extraocular dysfunction, lagophthalmos, corneal reflex impairment, as well as other similar neurological symptoms, such as papilledema [11].

Dysfunction of the oculomotor nerve can occur due to lesions between the midbrain and the orbit anywhere along its path. The third cranial nerve has both motor and parasympathetic components. The most typical causes of isolated third cranial nerve palsy are diabetes mellitus, posterior communicating artery aneurysm, and uncal herniation [12]. The unusual 
association between isolated third cranial nerve palsy and lymphoma was reported only in 22 cases upon literature review.

In 18 cases, the types of cells were described, and the most common phenotypes were large B-cell lymphoma [13]. BL was reported in only 4 of these cases ( 3 of them had HIV or immunosuppression-related lymphoma while only one case occurred in the immunocompetent patient). There was only one instance of oculomotor nerve paralysis due to Hodgkin's lymphoma [14].

Some authors documented similar cranial neuropathies associated with lymphomas in the CNS, but these neurological lymphomas are also associated with other clinical features of lymphomas (namely B-symptoms) or lymphadenopaothy [15]. Usually, other patients with lymphoma have supporting results that favor lymphoma diagnosis, such as a rise in leukocyte count and mediastinal masses, lymphadenopathy which was absent in our case, that mask the diagnosis of lymphoma initially [16].

This case is unusual because of the presence of isolated third cranial nerve paralysis without any other physical exam or a lab test that could lead us towards a proper conclusion on the diagnosis. In this scenario, establishing an accurate diagnosis is critical to guide the management strategy for high-grade B-cell lymphoma.

\section{Method of Literature Review}

For the years 1966-2020, we searched the MEDLINE database from the PubMed website, using the main terms lymphoma, third nerve palsy, HIV, and leptomeningeal disease neurolymphomatosis, and AIDS. Further papers were derived from the retrieved articles through an analysis of the reference list. Non-English publications in literature have not been reviewed.

\section{Conclusion}

Isolated third cranial nerve palsy can be only the sign that leads to critical illness like BL. In the absence of other causes of isolated oculomotor palsy, keep an open mind to such a rare association between BL and third cranial nerve palsy because time management is crucial in establishing the correct diagnosis and starting management.

\section{Acknowledgement}

The authors would like to acknowledge the Internal Medicine Residency Program for scientific support.

\section{Statement of Ethics}

This case was approved by the Hamad Medical Corporation's Medical Research Center, written informed consent was obtained from the patient and his family for publication of this case report and any accompanying images.

\section{Conflict of Interest Statement}

The authors have no conflicts of interest

\section{Karger's}




\section{Case Reports in Oncology}

Case Rep Oncol 2021;14:1555-1560

\begin{tabular}{l|l}
\hline DOI: $10.1159 / 000514983$ & $\odot 2021$ The Author(s). Published by S. Karger AG, Basel
\end{tabular} www.karger.com/cro

Abdelmahmuod et al.: Oculomotor Cranial Nerve Palsy with Burkitt's Lymphoma

\section{Author Contributions}

Elabbass A. Abdelmahmuod: writing, editing, and literature review. Mohammed Abdulgayoom: writing and literature review. Mohammed Elhadi: writing and editing. Hiba Magboul: writing and editing. M.Z. Sharaf Eldean: imaging review. Sali Alatasi: imaging review.

\section{References}

1 Bhatti MT, Schmalfuss IM, Eskin TA. Isolated cranial nerve III palsy as the presenting manifestation of HIVrelated large B-cell lymphoma: clinical, radiological and postmortem observations: report of a case and review of the literature. Surv Ophthalmol. 2005;50(6):598-606.

2 Baartman BJ, Adamopoulou C. Isolated abducens palsy heralding occult human immunodeficiency virusrelated lymphoma. Neuroophthalmology. 2018 Jan 2;42(1):31-4.

3 Kalantri SA, Nayak A, Datta S, Bhattacharyya M. Isolated third nerve palsy: a rare neurological presentation of Burkitt's lymphoma. BMJ Case Rep. 2017;2017:bcr2017219670.

4 Isolated cranial nerve III palsy as the presenting manifestation of HIV-related large B-cell lymphoma: clinical, radiological and postmortem observations: report of a case and review of the literature. PubMed [Internet] [cited 2020 Dec 30]. Available from: https://pubmed.ncbi.nlm.nih.gov/16263372/.

5 Taga A, Russo M, Florindo I, Pavesi G. Isolated third cranial nerve palsy leading to the diagnosis of disseminated burkitt lymphoma. Neurologist. 2017;22(5):182-5.

6 Khaleefah MM, Narayanan S, Al Dallal HA, Jones CM, Friedland RP, Palade AE, et al. Isolated oculomotor nerve palsy as a manifestation of diffuse large B cell lymphoma: a case report. Oncol Lett. 2020 Sep 23;20(6):285.

7 Sato H, Hashimoto T, Yoneda S, Hirabayashi K, Oguchi K, Higuchi K. Lymphoma as a cause of isolated oculomotor nerve palsy. J Clin Neurosci. 2011 Sep;18(9):1256-8.

8 Meireles J, Garrett MC, Abreu P. Isolated III cranial nerve palsy: a Hodgkin's lymphoma? BMJ Case Rep. 2014 [cited 2020 Dec 30]. Available from: https://pubmed.ncbi.nlm.nih.gov/24759607/.

9 Belkacemi Y, Sio TT, Colson-Durand L, Onal C, Villà S, Anacak Y, et al. Primary extranodal lymphoma of the glands. Literature review and options for best practice. Crit Rev Oncol Hematol. 2019;135:8-19.

10 Good W, Lama P, Barile GR. Current ophthalmology and medicine. Surv Ophthalmol. 2005;50:607-8.

11 Mayer RR, Frankfort BJ, Strickland BA, Debnam JM, McCutcheon IE, Groves MD, et al. Leptomeningeal metastases presenting exclusively with ocular disturbance in 34 patients: a tertiary care cancer hospital experience. J Clin Neurosci. 2017 May 1;39:151-4.

12 Isolated III cranial nerve palsy: a Hodgkin's lymphoma?: PubMed [Internet] [cited 2020 Dec 30]. Available from: https://pubmed.ncbi.nlm.nih.gov/24759607/.

13 Taga A, Russo M, Florindo I, Pavesi G. Isolated third cranial nerve palsy leading to the diagnosis of disseminated burkitt lymphoma [Internet]. Neurologist. 2017 [cited 2020 Dec 30];22:182-5. Available from: https:// pubmed.ncbi.nlm.nih.gov/28859023/.

14 Vaphiades MS, Roberson GH. Imaging of oculomotor (third) cranial nerve palsy. Neurol Clin. 2017;35:101-13.

15 Brewington D, Petrov D, Whitmore R, Liu G, Wolf R, Zager EL. De novo intraneural arachnoid cyst presenting with complete third nerve palsy: case report and literature review. World Neurosurg. 2017 Feb 1;98:873-e31.

16 Isolated third cranial nerve palsy leading to the diagnosis of disseminated burkitt lymphoma: a case report and literature review: PubMed [Internet] [cited 2020 Dec 30]. Available from: https://pubmed.ncbi.nlm.nih. gov/28859023/. 